\title{
Editor's Introduction for Volume 6, Issue 1
}

It is the sixth year since we founded this international journal as an official publication of Society for Risk Analysis China (SRA-China, http://www.sra.org/china-sra). The journal is designed to promote a higher level of methodology and practice, and provide an international platform for sharing theory and applications of Risk Analysis (RA) and Crisis Response (RC). Particularly, it is considered as a journal in science and technology rather than policy and law related to risk issues.

This issue contains 6 papers. There are 3 contributions written in English and 3 contributions in Chinese with English abstracts. The papers can be divided into four categories: experimental riskology, risk perception, financial risk, and public security.

Based on the thinking of experimental riskology, the paper "An Approach to Ranking Integrated Models for Risk Assessment by Using the Internet of Intelligences” , by Wang and et al., built an Internet of intelligences to play the gobang game for ranking the analytic hierarchy process, the median method corresponding to time and comprehensive evaluation method, according their scores. The results show that for gobang, the effect of simple comprehensive evaluation method is better than analytic hierarchy process.

There are two papers in risk perception. The first paper "Eutrophication Hazard Evaluation Using Copula-Cloud”, by Liu, Wang and Wang, proposes a copula-cloud to model the randomness, fuzziness and dependence of each risk criterion in assessment. The suggested technique has been applied on cases of representative lakes or reservoirs in China. Results in comparison with relevant proposed methods verify the completeness and effectiveness of the technique. The second paper “High-rise Building Group Regional Fire Risk Assessment Model Based on AHP”, by Zhu and You, puts forwards a regional fire risk assessment model for high-rise building group considering the fire danger degree, basic characters of the high-rise building group and fire control capability Based on Analytic Hierarchy Process (AHP). The impact factors, values of typical risk parameter and the weight of each index are analyzed. An assessment program is further developed using Matlab, to perform the risk assessment procedures. The model is then applied to the regional fire risk evaluation of a high-rise building group.

There are two papers in financial risk. The first paper "Research on the Performance Evaluation of Government Venture Capital Fund Based on Factor Analysis and DEA Model”, by Zhang, Chen and Wang, analyzes and determines the evaluation index system relevant in the performance evaluation of government venture capital fund. The suggested system could help us to effectively evaluate the performance of the guidance fund. The majority of the fund operation used in this paper is comprehensive and effective. The second paper "Economic Fluctuations and Insurance Industry Crisis: the History, Status Quo and the Future”, by Guo, analyzes the reasons and mechanisms of insurance industry crisis in Japan and Taiwan, found the insurance industry crisis closely related to the economic fluctuation, and provided some warnings and references to the insurance industry of China.

There is a paper in public security, "The Effects of the Social Context on Pre-Decisional Processes of Protective Action in Beijing Communities”, by Liu, Zhang, Li and Li, which examined the effects of the social context on pre-decisional processes of individual protective action. A two-stage survey was carried out aimed at residents in Beijing communities in half a year. Main results are : (1) Media communication media communication activities can influence individual reception of risk information; (2) Face-to-face communication activities can influence individual attention behavior; (3) Friendly and kinship network does not influence any pre-decisional processes variable, but it can influence face-to-face communication instead.

We sincerely hope our reader will find this issue's information on RA and CR interface useful. Thanks to the referees for their strong support and kind help. And also thank the authors very much for all their outstanding contributions.

Editor-in-Chief:

Prof. Chongfu Huang

Email: hchongfu@126.com
Publication Chair of SRA-China:

Prof. Mu Zhang

Email: rim_007@163.com
Director of Editorial Department:

Prof. Junxiang Zhang

Email: jracr_srachina@126.com 